\title{
HIGHLIGHTS
}

IBD

\section{Prostaglandin E2 inhibits fibroblast migration in intestinal wound healing}

Florian Rieder and colleagues have demonstrated that prostaglandin E2 (PGE2) inhibits fibroblast migration in intestinal wound healing in patients who have IBD.

Impaired wound healing is sometimes found in patients with IBD and this can lead to complications such as fistula or stricture formation. "There is a striking imbalance between this large clinical problem and the lack of information available [on the] pathophysiology of intestinal wound healing," explains Rieder.

Migration of colonic lamina propria fibroblasts (CLPFs) to the site of inflammation is important for wound healing in patients with IBD. Several molecules are known to modulate this migration, but Rieder and colleagues decided to investigate whether PGE2 (which is increased in the intestinal mucosa of patients with IBD) also has a role. They used migration assays, immunoblotting, immunocytochemistry and enzyme-linked immunosorbent assay to investigate the effect of PGE2 on CLPF migration in primary cell cultures from healthy controls and patients with Crohn's disease.

Their results demonstrate dosedependent inhibition of CLPF migration by PGE2. "This is the first time this has been shown in the intestine," says Rieder.

The researchers speculate that this inhibition of CLPF migration might be a factor in impaired intestinal wound healing in IBD. "Critical topics to be addressed in the future are the development of in vivo systems to test how and to what extent fibroblast migration influences intestinal wound healing," concludes Rieder.

Isobel Franks

Original article Rieder, F. et al. Prostaglandin E2 inhibits migration of colonic lamina propria fibroblasts. Inflamm Bowel Dis. 16, 1505-1513 (2010) 